twelve years old. When I first saw her, she was in bed, suffering from what appeared to be a sharp feverish cold. The following morning, there was pain in the joints of one limb; and, by the evening, painful swelling in the joints of both the upper and lower extremities, such as left no doubt as to the nature of the complaint. Indeed, late in the evening of this second day, the joints of the fingers and toes were all affected. I immediately ordered for her eight pills-one to be taken every three hours-each containing a grain and a half of calomel, with a quarter of a grain of opium. In about twenty-four hours from this, an aperient mixture was prescribed, consisting of Epsom salts half an ounce, wine of colchicum a drachm, an ounce and a half of the compound tincture of senna, with cinnamon water to six ounces; of which an ounce was given every fourth hour to cause free purging. When this had been accomplished, and the fever and pain much reduced, the following was ordered.

Quinine, xxiv grains; dilute sulphuric acid, xx minims; red cinchona bark (in powder) $3 \mathrm{vj}$; and water to $\xi \mathrm{vj}$.

Of this, well shaken up, was given, perseveringly, a teaspoonful every three hours by day, and also in the night, when she was awake. Before the end of the eighth day, the pain had left; and in a fortnight she might be said to be quite well. This case occurred about two years ago, and the health has ever since been sound and vigorous. Last autumn, I treated a strong girl of eleven, living in the country, in the same manner as the above; and she had to keep her bed only for nine or ten days, and has since been in excellent health.

It is assumed, when I am speaking of the treatment of acute rheumatism, that the patient is seen by me at the commencement. When a case has been going on without treatment, it may be, for two or three weeks, the remedies I have mentioned might not be altogether applicable.

Certain important particulars, with reference to the general management of this disease, I give in the following order.

1. Be the patient ever so robust, and the fever and pain severe, I never bleed, either by the lancet or with leeches.

2. I avoid diaphoretics; that is, doses of the Dover's powder to cause and keep up profuse sweating; which was, I may say, the universal practice in my early days.

3. I forbid the use of fomentations to the inflamed joints ; indeed, I forbid every kind of application to the joints, excepting dry warm cotton, or a dry warm silk handkerchief. The use of friction with some anodyne liniment (apt to be had recourse to by the friends of the patient, unless guarded against) I also prohibit.

4. In patients under the age of twenty, I do not allow even the hands or face to be sponged or washed during the fever; indeed, I dread every kind of liquid application. I well remember, early in my residence here, a fine young man, about the age of sixteen, brought home from a boarding-school with acute rheumatism, his knees enveloped in wetted bandages. The consequence was death in three days, from cardiac inflammation.

5. The quinine and bark should not be given until the feverish symptoms have been allayed by the use of calomel and opium, followed by a purgative.

I will just add that, when the patient has been seen early, and treated as here recommended, I have never, during the last thirty years, known heartaffection to follow in a single instance.

I may state, in conclusion, that I am quite aware therc are several eminent authors who recommend, 660 in rheumatic fever, other remedies and modes of treatment than what I have mentioned; but I have thought it best, for the sake of brevity, to give an outline merely of my own views and experience.

\section{ON DIPHTHERIA.}

By J. West Walker, M.B.Lond., Spilsby, Lincolnshire.

[Concluded from p. 386.]

General Treatment. Chlorate of Potash. This medicine must be a special favourite in the treatment of diphtheritic diseases ; for, no matter what else be recommended, it invariably receives favourable mention. The many points of resemblance between the diphtheritic false membrane and the white patches of thrush and its allied diseases, naturally suggest the idea of their being analogous morbid products; and any remedy known to possess a marked influence over the one was likely to be introduced as applicable in the treatment of the other. The result is that, whenever the diphtheritic sign presents itself, it has become a matter of custom to resort to chlorate of potash. The action of this medicine is involved in obscurity. "It does not appear to undergo any chemical change in its passage through the system; a fact fatal to the hypothesis of the chemico-physiologists, who fancied that it gave oxygen to the system, and was, therefore, well adapted to patients affected with scorbutic conditions, which were supposed to depend on a deficiency of that principle." (Pereira.) Yet, notwithstanding all this, like many other articles of our materia medica, its use is pretty well recognised and its employment understood, though its modus operandi may not admit of ready explanation. It is generally considered to be one of the so-called salines ; to possess cooling, diuretic, febrifuge powers; and to have a similar action to the nitrate, though, according to my experience, in accomplishing its effects it is vastly inferior to the last named salt. In the treatment of foul nlcerated conditions of the parts within the cavity of the mouth, ample experience confirms its value; and, under such circumstances, its beneficial action may be relied on.

Nitrate of Potash is an older and better established fever medicine, while its use in cases of inflamed states of the mucous membrane of the faucial region has long been acknowledged.

Speaking summarily, then, chlorate of potash is the special remedy in diphtheritic affections of the mouth, and is of secondary importance in feverish states of the general system. Nitrate of potash is more applicable to affections of the throat in sthenic cases, and is one of the best febrifuges we possess.

Guaiacum. At the close of Dr. Watson's lecture on Cynanche Tonsillaris, he says: "I ought perhaps to add, that recently $\mathrm{Mr}$. J. Bell of Barhead has very strongly recommended the internal administration of powdered guaiacum in large doses, as being almost a specific in the case of cynanche tonsillaris. He gives as much as half a drachm, suspended by means of mucilage in a draught, every six hours. Mr. Bell has no doubt that the remedy, if timely administered, will cut the disease short in ninety-nine cases out of a hundred. It has been found successful in other hands also." I have not had an opportunity of consulting Mr. Bell's contribution; but have had ample means for testing the efficacy of his remedy. It has long been my custom to prescribe it with confidence and satisfaction in the treatment of cases of ordinary sore-throat; and I have no hesitation in recording my belief that, under such circumstances, it possesses a very decided influence for good. I was, conse- 
quently, early induced to resort to it in diphtheritic affections; and, according to my experience, the best means of general medical treatment of epidemic sore-throat with diphtheritic complications will be found in the judicious employment of this medicine.

Ammonia. The London Pharmacopœia furnishes us with a preparation, the ammoniated tincture of guaiacum, wherein guaiacum and ammonia are in happy combination - a preparation admirably adapted to affections of the throat in connection with general asthenic conditions; in a word, the desideratum in the treatment of diphtheria. I generally suspend the guaiacum by means of honey, and give it in the proportion of half a drachm of the tincture to an ounce of water. Of this mixture, from a tea- to two tablespoonfuls are given every hour or every four hours, according to the age of the patient and the severity of the case. In sthenic conditions, the remedy admits of admixture with nitrate of potash; while, when asthenia prevails, excess of ammonia may be added. Independently of the part which ammonia plays in the preparation just alluded to, it is of itself worthy of being considered one of the most serviceable of remedies in the treatment of diphtheritic diseases. It has long enjoyed the reputation of being the best stimulant we possess in adynamic states of the general system. In the low fevers, and wherever the heart's action shows signs of feebleness, this remedy, either in solution or in a state of vapour, must always be employed. But, more than this; of late, ammonia has been introduced as possessing special powers over the fibrine of the blood-checking its formation and promoting its solution; and, on these grounds, according to Dr. Richardson, in affections accompanied with excessive formation and separation of fibrine, it is to be looked on in the light of almost a specific. In the treatment of scarlet fever, Dr. Witt and others speak most highly in its favour; th ough here, again, I prefer it in the preparation of guaiacum before allnded to.

The Tincture of Perchloride of Iron takes a deservedly high place among the numerous remedies brought forward as applicable to the treatment of diphtheritic affections; for, when administered in answer to suitable indications, it is undoubtedly gifted with potent virtues. It has now fully establishod its claim to be considered the best therapeutical agent we have, when properly administered, in the management of erysipelas. And the nearer any diphtheritic case shall approach in its characteristics those of that disease, the more confidently can its beneficial action be relied on. In closely packed, badly drained, badly ventilated districts of large towns, we look for erysipelatous affections more than in the open country; and in such localities, it is, I doubt not, that this remedy, first introduced by Dr. Heslop in his practice amongst diphtherial cases at Birmingham, will be found more specially indicated and more generally successful. It has also long been known to be particularly applicable to certain affections of the kidney, and to have a very decided controling power over albuminuria, when occurring in the course of general diseases. Purpura, too, is a symptom which calls for its administration in suitable combinations. Again, knowing the intimate relationship between erysipelas and some forms, at least, of puerperal fever, this is the medicine we should be most inclined to trust in any case of the latter disease putting on the diphtheritic character. Lastly, I make it a rule of practice, to prescribe this preparation in the convalescent stages of all diphtheritic affections, and to continue its action for some time; for not only is it a most useful tonic and assistant toward recovery, but also, as $I$ am inclined to think, a powerful prophylactic against sequelæ. From what has been said, it follows that, whenever the diphtheritic sign occurs in connection with erysipelas, erysipelatous affections, or puerperal fever-whenever, in a diphtheritic case, albuminuria or purpura manifest themselves-and whenever we require a tonic to assist convalescence, or to ward off secondary affectionsthe tincture of perchloride of iron becomes a remedy worthy of special commendation.

Iodine and the Iodides. In chronic enlargements of the tonsils, a condition particularly predisposed to assume the diphtheritic character, the iodide of iron, with cod-liver oil, becomes the most suitable remedy; while the syphilitic throat-and I have known bad and obstinate cases of so-called diphtheria to be nothing more than this disease complicated-requires, if to be satisfactorily treated, the employment of the iodide of potassium. The diphtheritic syphilitic affection, like erysipelas, is, doubtless, more frequently met with in urban than in rural practice; and, consequently, the practitioners of our cities will be more constantly called upon to resort to this particular remedy in the treatment of diphtheria than we of the country. "Dr. Willoughby Wade claims great powers for the iodide of potassium as a diphtherial medicine. $\mathrm{He}$ gives the following propositions: 1. "Assuming the presence of a substantive poison in the system, we know no drug which will act as a direct eliminant but iodide of potassium. It positively eliminates lead, and, we may presume, it positively eliminates syphilis." 2. "I employ iodide of potassium in two-, three-, or four-grain doses every two or three hours. I have been in the habit of conjoining with it chlorate of potash in five- or ten-grain doses." 3 . "I have known no instance of fatal termination where this plan of treatment has been carried out. I have known no instance of serious symptoms or of secondary paralysis supervening where this plan has been rigorously carried out."

Quinine may be employed as a general tonic in diseases attended with such often rapidly induced debility as is to be witnessed during a diphtheritic epidemic, though, to my mind, with very uncertain consequences. It is as a fever medicine, as an antiperiodic, rather than as a tonic, that its beneficial effects are displayed; and whenever the general disease puts on the intermittent character, and this is often the case in districts where ague is wont to abound, quinine and arsenic become our sheetanchors.

The Mineral Acids are, perhaps, as useful as anything in the treatment of diphtheritic complications of the relaxed throat; and, according to Dr. Chambers and those who go with him, they furnish us with the best medicine for cases of the continued fevers.

Calomel. Much-and, to my thinking, unnecessary - fear has prevailed on the subject of the use of calomel as a diphtherial medicine. It behoves us to study well our disease; to be fully alive to its asthenic character; and to exercise due caution in the administration of depressants ; but, on the other hand, let us beware lest our caution become overcaution, and we neglect to employ means of benefit to our patient. A smart purge of calomel, in connection with rhubarb, jalap, etc., when indicated, will have a rectifying influence on disordered secretions; it will assist in eliminating materies morbi, and often cut short the disease. In diphtheritic croup, calomel is to be administered in like manner as in croup not so complicated. The usual emetics and expectorants having been tried, a small dose of calomel every hour, until one of its physiological effects, the chopped spinach evacuations, be produced, has in my hands proved a successful mode of treatment, and one to which we must not be afraid to resort.

661 
From this survey, rapid and meagre though it be, of the different medicaments which have been brought forward as applicable to the treatment of diphtheria, we are enabled to see, firstly, that each one may become the most suitable remedy, according as certain states of the system shall indicate; and secondly, that the study of the proper place and relative value of such general remedies, which, by continuing to look upon diphtheria as a single disease, must remain a matter of the greatest perplexity, becomes, by substituting the view of its complex nature, a matter of the greatest simplicity.

Now and then epidemics of miasmatic disease come amongst us in such a terrible shape, that curative treatment, be it ever so rational, seems completely powerless. No matter how well we think, how judiciously we act, patient after patient succumbs; and we are driven, sorrowfully yet honestly, to confess the total inability of medicines to cope with the destroying influence. Some cases are so rapidly fatal as to give no time for the sufficient introduction of remedies; and others, owing to the parts concerned in the function of swallowing being implicated, admit not, it may be, of their suitable administration. The true explanation, however, of our want of success, lies probably deeper than in reasons such as these; and is to be found in the conviction daily growing upon us that therapeutic agents are not, especially in diseases of this class, to be looked upon as directly curative; but rather only as adjuvants and amongst the means of ordinary treatment. So long as we confine medicines to their proper places, expecting from them only what they can possibly perform (and much it is, I grant), we shall not be disappointed; but if we invest them with mysterious powers for good, which they certainly do not, and possibly it is not according to the nature of things that they ever can, possess, we not only lay ourselves open to bitter disappointment, but bring discredit on our art. When curative treatment becomes so unsatisfactory, it is well to turn our attention to preventive. Diphtheritic diseases are known "to manifest a decided tendency to be restricted to particular places, and to cling, as it were, to particular houses." (Greenhow.) 'The dwellers on one side of a river, in one village in a district, in one or two not necessarily contiguous houses in a village, are not unfrequently the only ones affected. The diseases resemble in this particular certain vegetable blights; which, as we know, will appear in one farm in a neighbourhcod, one field in a farm, or even one furrow in a field. This peculiar feature at once suggests a preventive remedy; which the confused notions prevalent on the subject of contagion have hitherto prevented from being carried out as fully as it deserves to be ; viz., the prompt removal of at least the unaffected from infested to non-infested localities. Nor need we, in order to accomplish this, always go far ; for I firmly believe I have seen the disease prevented in the unaffected, and stayed in the affected members of a family, by removing them no further than across the road.

Sequela. Any contribution on the subject of diphtheria must be considered incomplete which leaves unnoticed the secondary affections, proved by experience liable to affect the diphtheritic patient. "A peculiar character of the voice, resembling that produced by affections of the throat in secondary syphilis, is a common result of diphtheria, and often continues for many weeks after recovery. The power of swallowing is sometimes so impaired, that there has been difficulty in sustaining life during convalescence, and liquids especially are apt, even after a comparatively slight attack, to be regurgitated through the nostrils. Extreme anæmia, impairment of vision, a pe- culiar form of paraplegia, weakness of the hands and arms, numbness, tenderness of the limbs, tingling, wandering pains, and, more rarely, nervous sequelæ of a hemiplegic character, are, in the order here written, ulterior consequences of diphtheria. Gastrodynia, and sometimes dysenteric diarrhœa, occasionally follow diphtheria. Pain of the ear, deafness and abscess, are occasional but rare results of the disease." (Greenhow.) Add to these sudden, often fatal, syncope, and a more or less persistent feebleness of the heart's action, and we have a tolerably complete epitome of the diphtheritic sequelæ. By those who hold the specific disease theory, these secondary affections are considered to bear a special relation to one and only one primary affection; and in all definitions of diphtheria liability to such affections is included. I have previously attempted to show, that the diphtheritic formation - the pathognomonic of the supposed distinct disease-is merely a characteristic of an epidemic of many general diseases; and I venture to assert, that these secondary affections are quite as readily understood and explained by supposing them, in like manner, to belong to many rather than to one disease. They simply indicate the prior existence of a certain pathological condition; and such condition, whatever it be, may quite likely be the common property of many analogons diseases. At present, the special pathology of these sequelæ is clouded in obscurity. Some seem to indicate that the nervous system has received an influence capable of perverting its healthy function, though morbid anatomy is as yet quite unable to disclose alteration of structure, by which to account for such perversion of function. Others seem more readily interpreted by the light which has recently been thrown on pathology from the study of emboli and their relation to overfibrinated blood. And others, again, may perhaps be more properly classed as second affections taken on during the convalescent stage, the anæmia or spanæmia of such stage mainly predisposing thereto; after the primary disease, to which, too, they bear no regular proportion, either direct or indirect, quoad severity; and 2 , the only remedy found serviceable in treatment, change of air, becomes, by adopting this supposition, more easily understood. Further observation is, however, required, before the final settlement of this part of my subject; it is enough for my purpose, to state that hitherto no fact has been advanced in connection with the diphtheritic sequelæ, which cannot quite as readily be explained by the theory which considers them to be common to many diseases, as by that which connects them only with one.

Ancient Rome. "In Rome," says Galen, " no one seeks after truth; money, public offices and voluptuousness are the sole objects of life. He who devotes himself to the acquisition of knowledge, is considered insane. I am often blamed for applying myself too closely to researches after truth. They say that I will never gain any advantage, either for my friends or for myself, until I throw aside this habit, and pay my court to the great in the morning, and sup with them in the evening. It is thus, rather than by merit and learning, that confidence is inspired. And how can it be otherwise? Who are the judges between us? They are men who pass every hour of their lives in frivolous and disreputable occupations. When ill, they do not seek the attendance of the best informed physician, with whom, when in health they had no acquaintance; but they call in those who have been their boon companions, who flatter them; give them cold water, if they so desire; baths if they wish ice, wine, in a word, anything they fancy." 